\title{
Research Paper: The Effect of Zinc and Vitamin D Nutrition on Reducing Morphine Side Effects and Development of Dental Anomalies in Rats Newborns
}

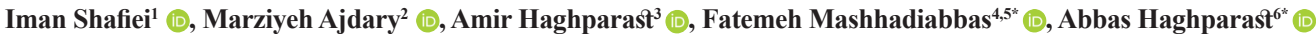

1. Department of Prosthodontics, Faculty of Dentistry, Kerman University of Medical Sciences, Kerman, Iran.

2. Cellular and Molecular Research Center, Iran University of Medical Sciences, Tehran, Iran.

3. School of Dentistry, International Branch of Shahid Beheshti University of Medical Sciences, Tehran, Iran.

4. Department of Oral \& Maxillofacial Pathology, School of Dentistry, Shahid Beheshti University of Medical Sciences, Tehran, Iran.

5. Dental Research Center, Research Institute for Dental Sciences, School of Dentistry, Shahid Beheshti University of Medical Sciences, Tehran, Iran.

6. Department of Physiology, Neuroscience Research Center, School of Medicine, Shahid Beheshti University of Medical Sciences, Tehran, Iran.

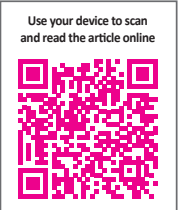

Crtation: Shafiei, I., Ajdary, M., Haghparast, A., Mashhadiabbas, F., \& Haghparast, A. (2020). The Effect of Zinc and Vitamin D Nutrition on Reducing Morphine Side Effects and Development of Dental Anomalies in Rats Newborns. Basic and Clinical Neuroscience, 11(4), 403-412. http://dx.doi.org/10.32598/bcn.11.4.38.6

http://dx.doi.org/10.32598/bcn.11.4.38.6

Article info:

Received: 17 May 2019

First Revision: 06 Jun 2019

Accepted: 13 Aug 2019

Available Online: 01 Jul 2020

Keywords:

Addiction, Morphine,

Minerals, Histological changes,

Incisor teeth, Rats

\section{AB S T RA C T}

Introduction: The use of opioids such as morphine has anti-pain effects along with some side effects on body organs. Opioids such as morphine can be transferred from mother to child through the placenta and or breastfeeding. This study aimed to assess the effect of morphine on mineral content and histological changes of incisor teeth of rats born to morphine-addicted mothers.

Methods: In this experimental animal study, 24 pregnant rats were randomly divided into 6 groups of control, morphine, zinc, vitamin D, morphine plus zinc, and morphine plus vitamin D. After completion of the breastfeeding period, two babies were randomly selected among the newborns of each mother rat. Mineral content was analyzed using the Rontec device. The obtained data were analyzed by Newman-Keuls multiple comparisons test in Prism 5.

Results: Results showed a significant reduction in fluorine content in the experimental groups compared with the control group $(\mathrm{P}<0.05)$. The magnesium content in the experimental groups was significantly higher than that in the control group $(\mathrm{P}<0.05)$. Microscopic assessment of the slides showed a significantly less enamel maturation in the experimental groups compared with the control group $(\mathrm{P}<0.05)$.

Conclusion: Morphine use by mothers decreased the fluorine content of tooth structure and retarded the maturity of the enamel of infants.

\section{* Corresponding Author:}

Fatemeh Mashhadiabbas, PhD.

Address: Department of Oral \& Maxillofacial Pathology, School of Dentistry, Shahid Beheshti University of Medical Sciences, Tehran, Iran. Tel: +98 (21) 2243-1624

E-mail:fmashhadiabbas@yahoo.com

\section{Abbas Haghparast, PhD.}

Address: Department of Physiology, Neuroscience Research Center, School of Medicine, Shahid Beheshti University of Medical Sciences, Tehran, Iran Tel: +98 (21) 2243-1624

E-mail:haghparast@yahoo.com; haghparast@sbmu.ac.ir 


\section{Highlights}

- Morphine use by mothers decreased the fluorine content of tooth structure and retarded the maturity of enamel of infants.

- Use of morphine has side effect on histological changes of incisor teeth.

- Morphine causes mineral loss and nutritional deficiencies which have a profound adverse effect on tooth structure and susceptibility to caries.

\section{Plain Language Summary}

Opioids to illegal substances is a complex health dilemma, causing a wide range of skeletal and dental complications, oral infections, and psychological disorders. Opioids such as morphine can be transferred from mother to child through the placenta and or breastfeeding. Morphine use by mothers decreased the fluorine content of tooth structure and retarded the maturity of the enamel of infants. Morphine's unique mechanism of action and its metabolic pathway suggest its correlation with tooth decay. This study showed that morphine can decrease the concentration of minerals, and prevent caries. Also, the administration of zinc and vitamin D can increase the absorption of fluorine, magnesium, zinc, iron, and strontium. Dentin thickness is increased with zinc and vitamin D supplementation.

\section{Introduction}

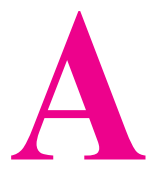

ddiction to illegal substances is a complex health dilemma, causing a wide range of skeletal and dental complications, oral infections, and psychological disorders (Chen $\&$ Lin, 2009). Substance abuse and its related complications impose a great burden on the individual and social health and can decrease work efficiency and production, increase health care costs, and higher violence and crime (Nessa, Latif Siddiqui, Hussain, \& Hossain, 2008). Oral health problems are among the most common general health complications associated with substance abuse. Substance abuse has direct and indirect effects on oral and dental health. Dental problems can adversely affect the quality of life of drug abusers (Robinson, Acquah, \& Gibson, 2005; Reece 2007). The use of opioids is associated with a high rate of caries and periodontal disease, which is significantly higher than the rate in the general population (Scheutz 1984). Drug abuse also causes mineral loss and nutritional deficiencies such as protein and vitamin A deficiency, which have a profound adverse effect on tooth structure and susceptibility to caries (Sebastiani et al., 2018). Navia, Di Orio, Menaker and Miller (1970) stated that zinc, as a rare-earth element, can prevent caries progression. Lin and Leo reported an increased rate of caries following the reduction in plasma level of zinc in rats under treatment with contraceptive steroids (Lin, Lin, Tsai, Lin, \& Chen, 2011). Zinc deficiency is also related to taste disorders (Larson, 1964) and parakeratosis of the buccal epitheli- um in laboratory animals (Navia et al., 1970). Zinc comprises a large portion of the enamel and is only supplied by eating zinc-containing foods (Navia \& Jm, 1972). Brown, Calhoun, Larson and Smith Jr (1979) showed that severe zinc shortage $(<1 \mathrm{ppm})$ in the nutritional regimen of mothers during breastfeeding period increased the susceptibility of their children's teeth to caries. In the past couple of decades, increasing attention has been paid to micronutrients and their role in human health and disease prevention. Calcium and vitamin D are among the most important micronutrients related to general health and caries prevention. Vitamin D is involved in calcium uptake and metabolism and has some other tasks in the human body as well. Calcium plays a role in bone health and is involved in many critical processes (Man et al., 2017). Calcium and vitamin D play key roles in the preservation of tooth structure. Due to their altered physiology and absorption of minerals and some pathological conditions, drug abusers have specific dietary needs. For instance, they often suffer from calcium and vitamin D deficiency. Consequently, dental caries is also prevalent in them. Attempts have been made to improve the nutritional status of drug abusers and they are encouraged to consume foods rich in vitamin $\mathrm{D}$, calcium and other micronutrients (Man et al., 2017). Vitamin D deficiency also impairs the absorption of several minerals and vitamins. Minerals such as iron, magnesium, zinc, and phosphate are affected by vitamin D shortage. Drug abusers often suffer from magnesium deficiency. Vitamin D deficiency decreases the absorption of magnesium and further aggravates the health status (Lhamo, Chugh, Gautam, \& 
Tripathi, 2017). Zinc is an import element for growth and development and its shortage causes dental caries. Substance abuse affects dental caries as well. Literature review yielded no comprehensive study on the simultaneous effect of drugs and nutrition on tooth structure. Considering the role of vitamin $\mathrm{D}$ in calcium uptake and the adverse effect of morphine on this process, this study aimed to assess the effect of vitamin D supplementation on mineral composition of teeth.

Although there is some information about the effect of morphine on oral and dental illnesses, no information is available on examining these changes separately in males and females. Considering the increased drug use among males and females in recent years, especially at the age of fertility, and lack of adequate and consistent information about the effect of morphine on dental structure, as well as its possible effects on newborns of these parents, the present study examines the effect of oral morphine consumption by pregnant rats on changes in incisors' mineral content, not reported in the available sources, and improvement of damages by vitamin $\mathrm{D}$ treatment.

\section{Materials \& Methods}

\subsection{Animals}

This experimental animal study was conducted on female Wistar rats weighing 200-250 g. Each rat was separately caged at $22^{\circ} \mathrm{C}-25^{\circ} \mathrm{C}$ temperature under $12: 12 \mathrm{~h}$ light:dark cycle with ad libitum access to food and water (Nessa et al., 2008; Chen \& Lin, 2009). Each rat was only experimented once. The rats were transferred to the lab one week earlier to be accommodated to their new environment. All experiments were performed following the guidelines of the National Institute of Health Publication No. 80-23, revised in 1996. The study was also approved in the Ethics Committee of Shahid Beheshti University of Medical Sciences, Tehran, Iran.

\subsection{Experimental design}

A total of 24 pregnant rats were divided into 6 groups as follows:

1. Control group: This group had no addiction to morphine during pregnancy or breastfeeding and did not receive zinc supplementation during the breastfeeding period.

2. Morphine group: This group was addicted to morphine during pregnancy and breastfeeding.
3. Zinc group: This group had no addiction to morphine during pregnancy or breastfeeding but received zinc during breastfeeding.

4. Morphine-zinc group: This group was addicted to morphine during pregnancy and breastfeeding and received zinc supplementation during pregnancy.

5. Vitamin D group: This group had no addiction to morphine during pregnancy and breastfeeding and received vitamin D supplementation during the breastfeeding period.

6. Morphine-vitamin D group: This group was addicted to morphine during pregnancy and breastfeeding and received vitamin D supplementation during the breastfeeding period.

\subsection{Addiction model}

To induce morphine dependency in mother rats, morphine was added to their drinking water for 21 days. Morphine was added in $0.1,0.2$, and $0.3 \mathrm{mg} / \mathrm{mL}$ concentrations each for 48 hours (a total of six days); 0.4 $\mathrm{mg} / \mathrm{mL}$ concentration of morphine was used for the remaining 15 days until the $21 \mathrm{st}$ day. Morphine administration was continued with a $0.4-\mathrm{mg}$ dose until the end of breastfeeding period (which was approximately three weeks) (Navia \& Jm, 1972). To mask the bitter taste of morphine, $0.3 \%$ sucrose was added to the drinking water of rats. To ensure addiction, one rat was randomly chosen from each group and received an injection of $2.5 \mathrm{mg} / \mathrm{kg}$ naloxone as a morphine antagonist. Witnessing signs and symptoms of withdrawal confirmed the addiction of rats.

\subsection{Treatment for zinc}

In zinc and morphine+zinc groups, mother rats received zinc sulfate supplementation during their breastfeeding period. The recommended daily dose of zinc was $12 \mathrm{mg} /$ $\mathrm{kg}$. Since mother rats weighed about $250 \mathrm{~g}$, they had to receive $3 \mathrm{mg}$ of zinc daily during their breastfeeding period. Zinc sulfate capsules contain $50 \mathrm{mg}$ of zinc. Thus, $3 \mathrm{mg}$ of zinc was measured by a digital scale and was added daily to the drinking water of mother rats and the process was continued until the end of their breastfeeding period (Friedler \& Cicero, 1987).

\subsection{Analysis of inorganic elements}

After completion of the breastfeeding period (21 days), two babies were randomly chosen among the newborns of each mother rat and decapitated. Of all upper and 
lower incisors of infants, one maxillary and one mandibular incisor were chosen for the analysis of the mineral content. Two adjacent teeth were randomly chosen for histological assessment and immersed in formalin for fixation and processing. A total of 48 specimens in the experimental and 16 in the control group were subjected to the analysis of mineral content and 16 specimens of the same teeth of the same animals were subjected to histological analysis. In mineral content analysis, the calcium, fluoride, zinc, magnesium, manganese, phosphorus, strontium, iron, and chlorine content in teeth were analyzed by X-ray diffraction spectroscopy in Razi Metallurgy Research Center and the weight percentage of these elements was determined using the Rontec machine (Germany) (Figures1 \& 2).

\subsection{Histology}

For histological assessment, the specimens were fixed in $10 \%$ formalin for 48 hours and were then decalcified in $10 \%$ formic acid for 20 days; $4-\mu$ longitudinal serial sections were made and stained with hematoxylin and eosin. The slides were then evaluated by a blind oral pathologist using Nikon (E400, Japan) light microscope. After the preparation of slides, dentin thickness was measured using IHMM software version 1.1 (SBMU, Tehran, Iran) at 40X magnification. Degree of enamel maturation (absence of matrix, presence of one-third of the matrix, presence of two-thirds of the matrix, presence of complete matrix) and pulp necrosis were deter- mined under a light microscope (E400, Nikon, Japan) at 40X, 100X, and 200X magnifications.

\subsection{Statistical analysis}

The obtained data are expressed as Mean \pm SEM (Standard Error of Mean). The data were analyzed by commercially available GraphPad Prism ${ }^{\circledR}$ 5.0. To compare the percentage of mineral, enamel maturation, and dentin thickness between all groups (vehicle and experimental groups), tow-way and or one-way Analysis of Variance (ANOVA) followed by post hoc student's t-test were used as needed. $\mathrm{P}$ values less than $0.05(\mathrm{P}<0.05)$ were considered to be statistically significant.

\section{Results}

\subsection{The effect of zinc and vitamin D nutrition on re-} ducing morphine side effects and mineral elements

Figure 3 shows the weight percentage of calcium in the teeth of rats born to the morphine group compared with the control group. The weight percentage of calcium in the experimental groups was almost equal to that in the control group with no significant difference. The weight percentage of calcium in the morphine-vitamin D and zinc groups were not significantly different from that of the control group. Figure 3 shows the weight percentage of phosphorous in the teeth of rats born to the morphine group compared with the control group. As shown, the weight percentage of phosphorus in the experimental groups was almost equal to that of the control group

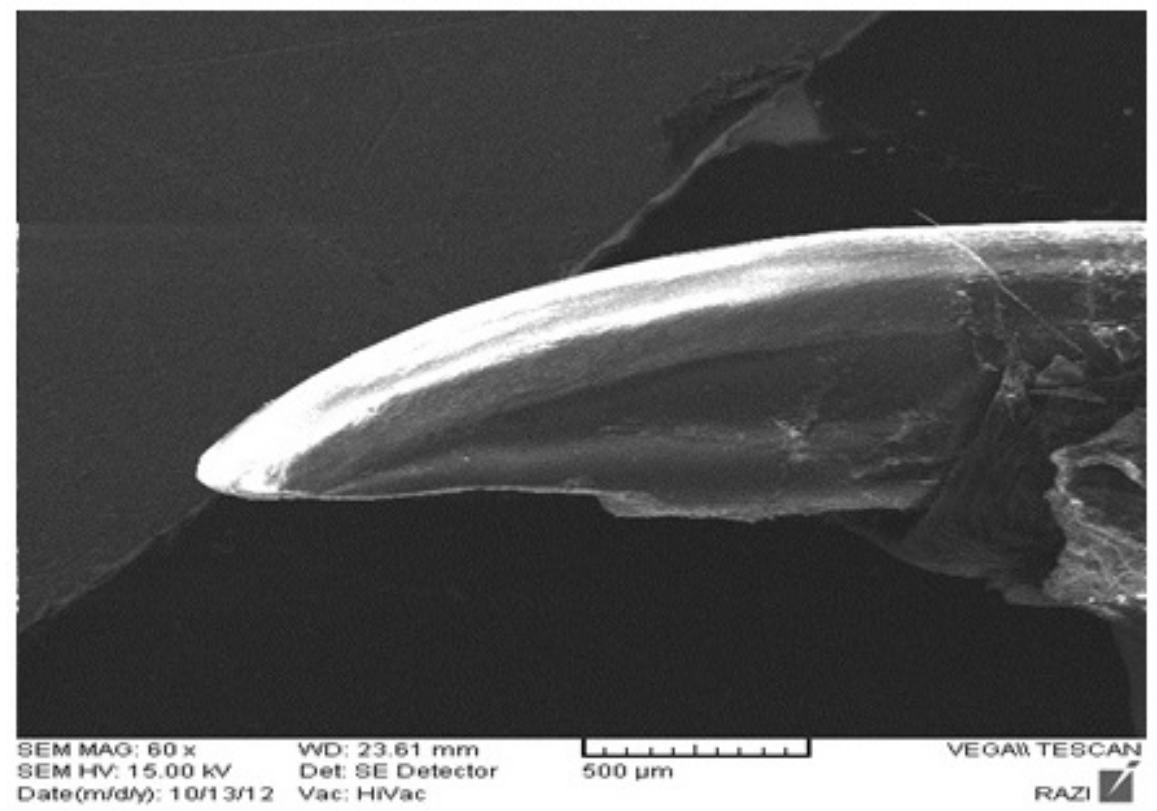

Figure 1. Electron microscopic image of incisor tooth of a rat 


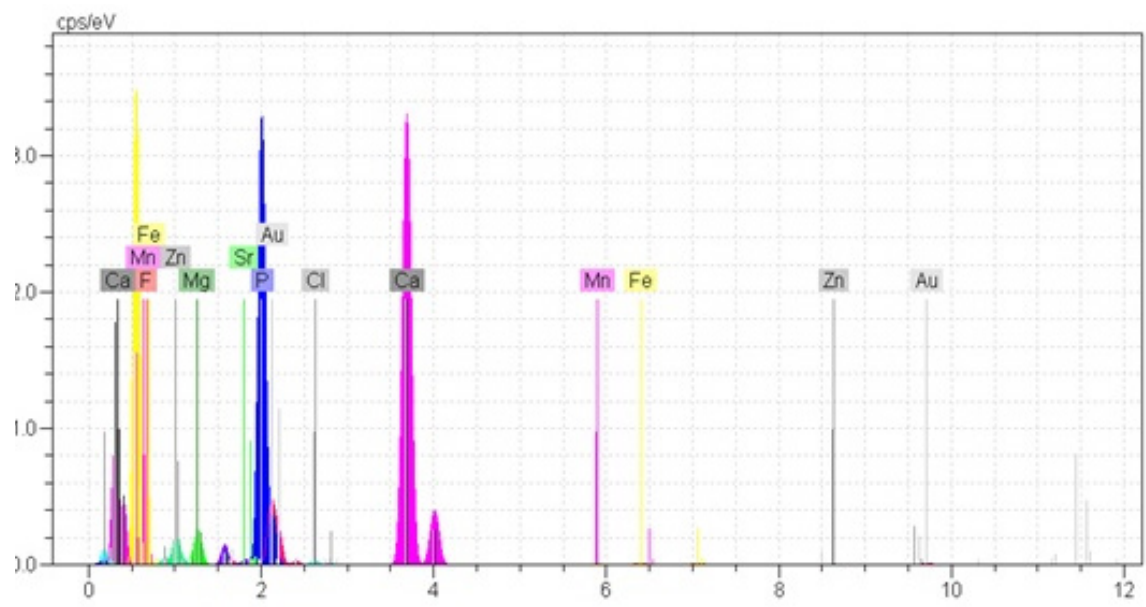

NEUR SCIENCE

Figure 2. Elements present in the structure of incisor tooth of a rat under electron microscopy using X-ray diffraction
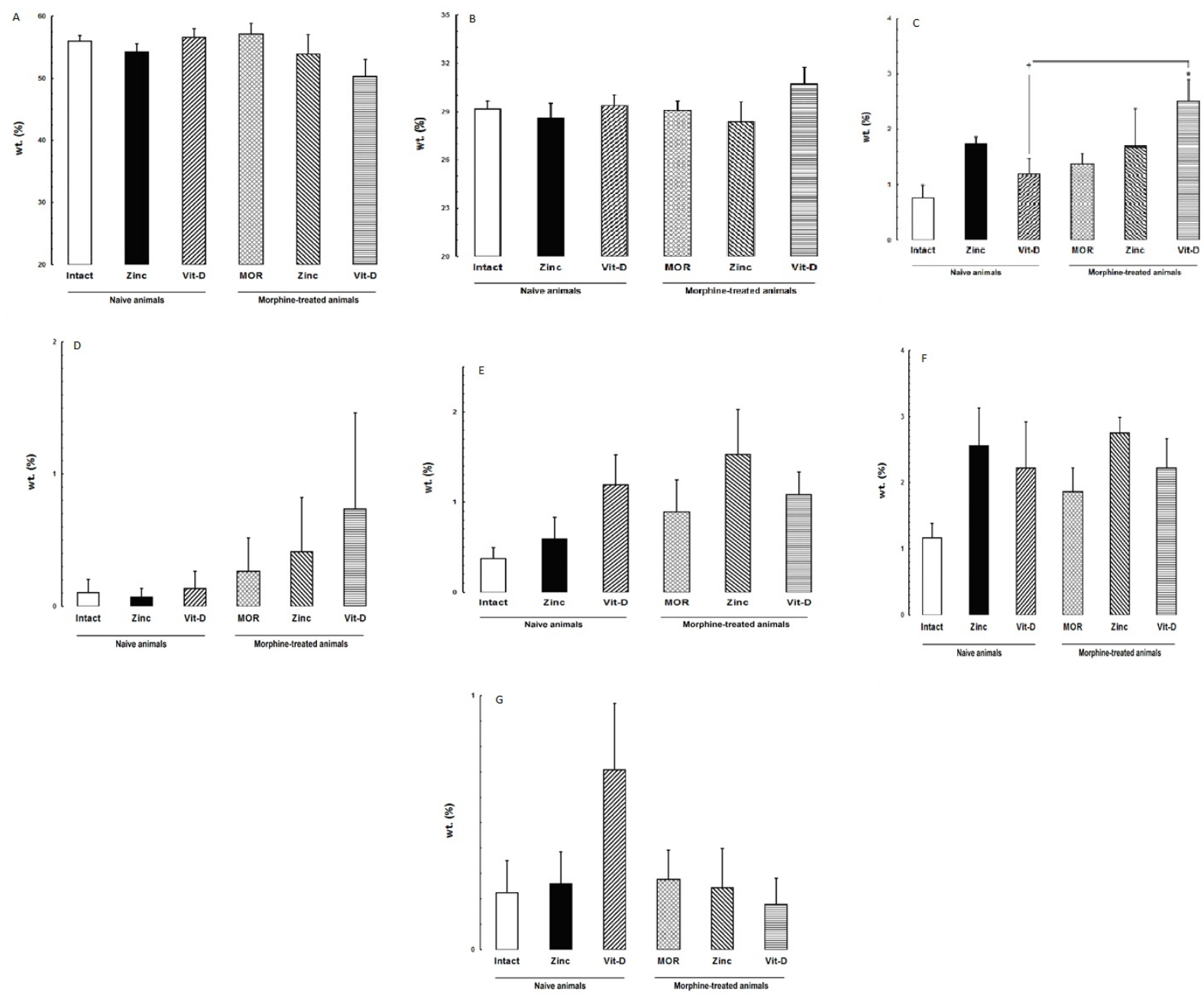

Figure 3. The weight percentage of mineral elements in teeth of rats born to morphine-addicted mothers compared with the control group

A: Ca; B: p; C: mg; D: Zn; E: fe; F: Sr; G: mn; * P<0.05 level of significance 

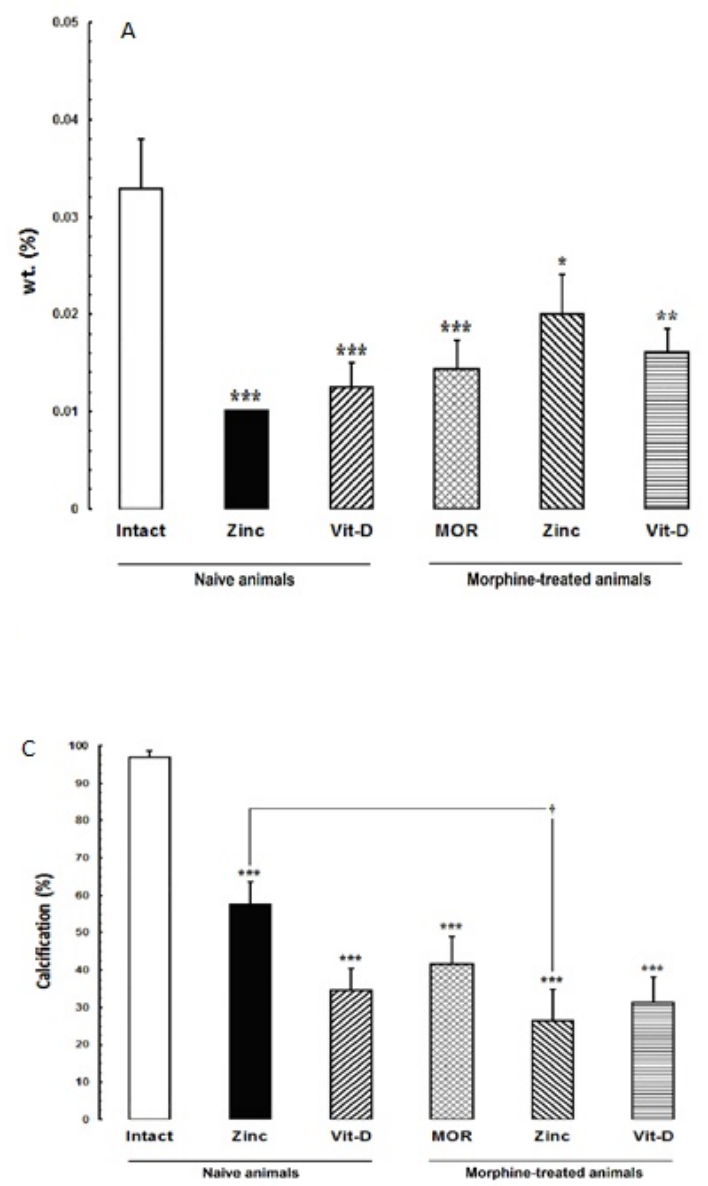
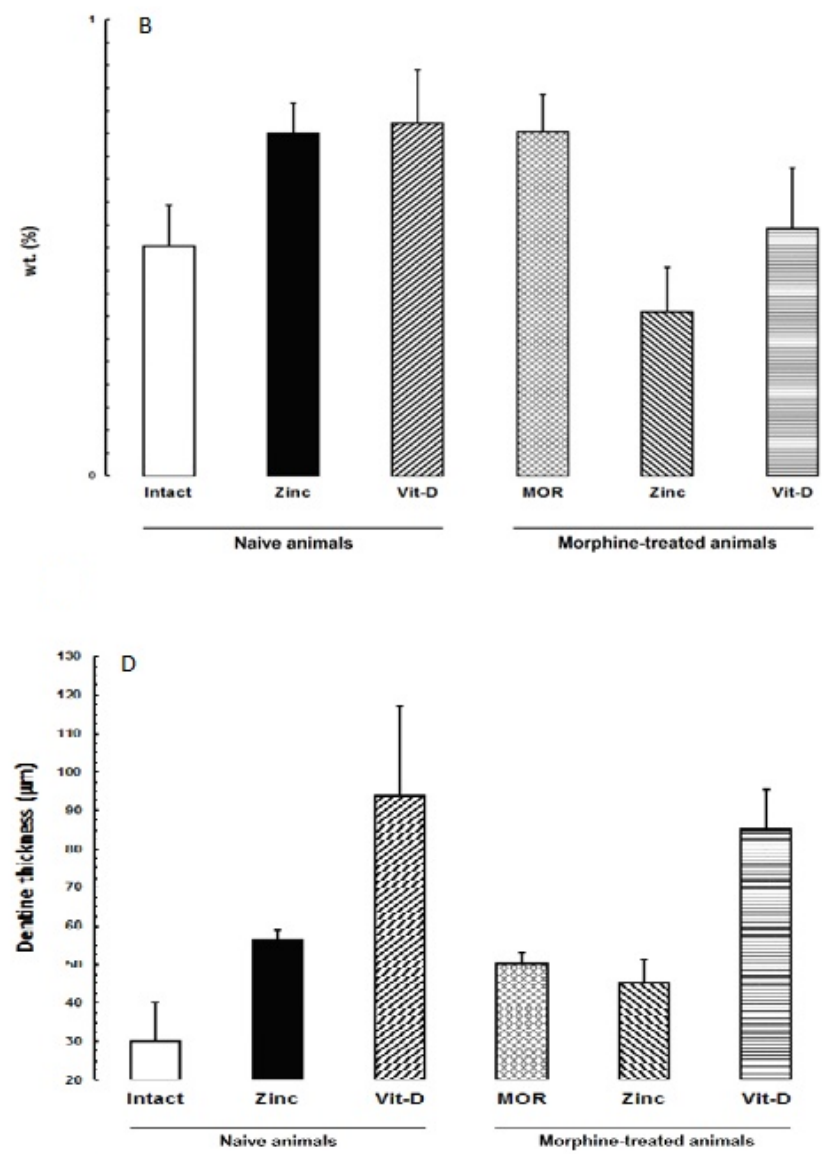

NEUR SCIENCE

Figure 4. The weight percentage of fluorine in teeth of rats born to morphine-addicted mothers compared to the control group A: F; B: Cl; C: Enamel maturation; D: Dentin thickness; ${ }^{*} \mathrm{P}<0.05$ level of significance

with no significant difference. The weight percentage of phosphorus was not significantly different in the morphine group compared with the control group. Vitamin D supplementation increased the weight percentage of phosphorus compared with the morphine group, but this increase was not statistically significant. An increase in the weight percentage of magnesium was noted in all groups compared with the control group but the increase was not statistically significant. A significant increase in the weight percentage of magnesium was only noted in the morphine and vitamin D group compared with the vitamin D control group. No significant difference was noted in the weight percentage of zinc between the experimental and the control groups. It increased slightly but not significantly in the morphine group. In the zinc control group (mothers receiving zinc supplementation during breastfeeding), the weight percentage of zinc slightly decreased (not significantly). In the morphinezinc group, the weight percentage of zinc was slightly higher than in the zinc group. No significant difference was noted in the weight percentage of chlorine between the experimental and the control groups. A slight, insignificant increase was noted in the weight percentage of zinc in all experimental groups except for the morphine and zinc plus vitamin D groups. No significant difference was noted in the weight percentage of manganese between the experimental and the control groups. In the healthy group, the administration of vitamin D increased the weight percentage of manganese while it reduced the weight percentage of manganese in the morphine group. No significant difference was noted in the weight percentage of manganese between the experimental and the control groups. However, a slight increase was noted in all experimental groups compared with the control group in this respect. Vitamin D and zinc supplementation increased the weight percentage of iron, and the effect of vitamin D was greater in this respect. No significant difference between the experimental and the control groups was noted in the weight percentage of manganese. However, in all experimental groups, the weight 
percentage of strontium increased slightly, but not significantly, compared with the control group.

\subsection{The effect of zinc and vitamin $D$ nutrition on reducing morphine side effects and tooth decay}

Figure 4 shows that the weight percentage of fluorine decreased significantly in all experimental groups compared with the control group, but it increased in morphine and zinc and morphine and vitamin D groups compared with the respective control groups. No significant difference was noted in the weight percentage of chlorine between the experimental and the control groups. A slight, insignificant increase was noted in the weight percentage of zinc in all experimental groups except for the morphine and zinc plus vitamin D groups. Enamel maturation rate was significantly lower in all experimental groups compared with the control group. Enamel maturation was significantly lower in the teeth of rats born to morphine group who received both zinc and vitamin D supplementation during their breastfeeding compared with the rats of healthy mothers who only received zinc supplementation during breastfeeding. However, dentin thickness in all experimental groups slightly increased compared with the control group. Vitamin D and zinc both caused an increase in dentin thickness, and vitamin $\mathrm{D}$ was more effective in this regard.

\section{Discussion}

The level of fluorine in the addicted group that received morphine along with zinc and vitamin D increased compared with the morphine-addicted group. In the case of magnesium, there was a significant increase in the addicted group treated with vitamin D as compared with the control group receiving vitamin D. Zinc level increased in the morphine-addicted mothers' group. There was a significant increase in the weight percentage of chlorine in all groups except for the morphine-addicted mothers' group that simultaneously received zinc and vitamin D during lactation. The weight percentage of iron was significantly higher in all of these groups than the control group. In other words, the administration of vitamin D and zinc can increase the level of iron while vitamin D is more potent in this regard. The weight percentage of strontium in all the groups increased insignificantly as compared with the control group. The degree of enamel maturity in all groups, whether those who had morphineaddicted mothers or those who had non-addicted mothers, decreased significantly as compared with the control group. A significant decrease in the degree of enamel maturity was observed in the teeth of newborns of morphine-addicted mothers who simultaneously took zinc and vitamin D during lactation as compared with that in newborns of non-addicted mothers who only took zinc supplement during lactation. There was no significant change in dentin thickness in any of the groups as compared with the control group $(\mathrm{P}>0.05)$. Vitamin $\mathrm{D}$ and zinc both increased dentin thickness in all groups, and the effect of vitamin D was stronger, but the increase was not significant for both.

Calcium and phosphorous are the two main constituents of dental hard tissue. X-ray diffraction for the assessment of change in the weight percentage of calcium and phosphorous showed that on average $50 \%$ of the minerals in teeth of baby rats were calcium and $30 \%$ was phosphorous. The results showed that the addiction of mothers to morphine did not cause a significant change in the weight percentage of calcium and phosphorous. Receiving zinc supplementation had no significant effect on the weight percentage of either calcium or phosphorous. Our results showed that the weight percentage of fluorine, which is crucial for caries prevention, was significantly lower in rats born to morphine-addicted mothers. The weight percentage of fluorine in zinc and vitamin D groups was still significantly lower than that of the control group, showing that zinc and vitamin D supplementation could not completely compensate for this shortage (Nadeau et al., 2018).

The weight percentage of strontium, iron, and magnesium increased in all experimental groups (morphine-addicted and healthy mothers) compared with the control group, but the situation was opposite for fluorine in a way that all experimental groups showed significantly lower weight percentage of fluorine compared with the control group. Regarding chlorine, it may be stated that zinc and vitamin D supplementation in the healthy group increased the weight percentage of chlorine, but the morphine group showed a reduction in the weight percentage of chlorine compared with its respective control group. The reason may be a low dose of supplements, low concentration, or short duration of their administration.

Histological analysis revealed a significantly lower maturation of enamel in the teeth of rats born to morphine-addicted mothers. An important finding of the current study was significantly lower enamel maturity in the teeth of rats born to morphine-addicted mothers who received zinc supplementation during breastfeeding compared with the rats born to healthy mothers who received zinc or vitamin D supplementation. In the current study, morphine addiction decreased the weight percentage of fluorine, which may be attributed to increased blood acidity (Gupta, 2018; Henkel, Altenburger, Au- 
wärter, \& Neukamm, 2018) and changes in blood carriers of elements resulting in decreased transportation and uptake of these elements by the mineral structure of teeth of rats born to morphine-addicted mothers. A previous study showed that the risk of osteoporosis was related to the chronic use of morphine by rats, but treatment with morphine can also cause osteoporosis (Rashidpour et al., 2012). They showed that morphine would decrease the weight percentage of calcium and compromise the tissues mainly composed of calcium (such as teeth). The inhibitory effect of morphine can be associated with decreased activity of osteopontin and osteocalcin (Rashidpour et al., 2012). When the process of regeneration is impaired and the mineral content (especially calcium) decreases, the activity of osteoblasts decreases as well.

Zinc and vitamin D were used for the treatment of calcium deficiency in this study but did not have a significant efficacy to compensate for calcium loss. The reason may be the dosage or duration of drug intake in which case changing these factors may reverse reduction in calcium level. The neutral effect of morphine on some mineral constituents of teeth is predictable considering the unique properties of morphine and its mechanism of action. In the current study, we also assessed the effect of morphine on teeth histologically and it was found that dentin thickness decreased due to morphine intake, which improved by the use of supplements. Sekhavat, Mousavizadeh, Pakshir and Aslani (2002) evaluated the histological effects of morphine on teeth and showed that it affected teeth movement. This justifies the histological effects of morphine on tooth structure.

According to previous studies, zinc supplementation can decrease fluorine in the serum and urine. Although the amount of sodium fluoride may significantly increase in hard tissues, this increase is negatively affected by the presence of zinc. The effect of zinc on fluorine can be used for the prevention of fluoride toxicity (Gupta, 2018). Lin et al. (2011) evaluated the effect of zinc chloride on odontogenesis by dental pulp stem cells through increasing metallothionein. They were the first to show that zinc chloride can induce differentiation of dental pulp odontoblasts by increasing the expression of the metallothionein gene. Thus, zinc can indirectly increase dentin formation, and this effect may be significant (Lin et al., 2011). A previous study assessed the relationship between zinc content of tooth structure and susceptibility to caries and reported that zinc shortage in the diet decreases the amount of zinc in the enamel and dentin structure and increases the susceptibility to caries (Cerklewski, 1981). The current study showed that zinc had an indirect effect on fluorine such that the weight per- centage of fluorine was significantly lower in rats born to mothers who received zinc supplementation compared with the control group. On the other hand, a reduction in weight percentage of fluorine in the teeth of rats born to morphine-addicted mothers who received both zinc and vitamin D supplementation during breastfeeding (compared with the control) highlighted the role of zinc and vitamin D nutritional supplements. Regarding changes in enamel maturation of teeth and considering the reduction in enamel maturation in morphine-addicted mothers, it seems that as morphine increases blood acidity, it can cause changes in calcium chelators and other carriers, leading to alterations in blood concentration and subsequently susceptibility to tooth calcification. Based on these findings, future studies should quantify the percentage of minerals in the hard tissue and also measure their serum concentration. The activity of major cells in the process of tooth mineralization such as ameloblasts and odontoblasts is measured by the expression of their markers. In their study on rats, Aghili et al. stated that the efficacy of tramadol for orthodontic teeth movement was dose-dependent (Aghili, Moghadam, Yassaei, Meybodi, \& Tabatabaei, 2013). Molecules produced in different tissues, drugs, and foods consumed by the patient can reach paradental tissues via the blood circulation and cell interactions. The combined effect of mechanical forces and one or more of these factors can be encouraging or inhibiting (Aghili et al., 2013). Patients referring to dentists may use a variety of medications, vitamin supplements, or minerals and these factors can affect the shortterm or long-term treatment outcomes. However, the risk of significant interactions between the factors affecting bone formation is very low (Gibson 1996).

Enamel is the most mineralized material in the human body. It is mainly composed of calcium and phosphate. Vitamin D increases the uptake of calcium and phosphate, which can strengthen the teeth. Vitamin D receptor is present on immune cells and teeth. Evidence shows that high levels of vitamin D during pregnancy and lactation can decrease the risk of dental caries in children. Fluoride induces osteoblastic proliferation and bone formation (Sekhavat et al., 2002). Fluoride reduction compromises bone formation, its remodeling, and tooth structure. Fluoride ions have an affinity for hydroxylcalcium crystals, leading to calcification of hard tissues, vessel walls, articular capsules, and ligaments (Gibson 1996). In the current study, no change was noted in the weight percentage of calcium.

Morphine's unique mechanism of action and its metabolic pathway suggest its correlation with tooth decay. This study showed that morphine can decrease the concentration of minerals, and prevent caries. Also, the administration of zinc and vitamin D can increase the absorption of fluorine, 
magnesium, zinc, iron, and strontium. Dentin thickness is increased with zinc and vitamin D supplementation.

\section{Ethical Considerations}

\section{Compliance with ethical guidelines}

All ethical principles were considered in this article.

\section{Funding}

This research did not receive any specific grant from funding agencies in the public, commercial, or not-for-profit sectors.

\section{Authors' contribution}

Wote the study protocol and organized the dental examination: Iman Shafiei; Performed the data entry and statistical analyses and finalized the manuscript: Abbas Haghparast; Designed the study, supervised the data analysis, and edited the manuscript: Fatemeh Mashhadiabbas; Developed the used measures and edited the manuscript: Marziyeh Ajdary; All authors read and approved the final version of the manuscript.

\section{Conflict of interest}

The authors declare no conflict of interest.

\section{References}

Aghili, H., Moghadam, M. G., Yassaei, S., Meybodi, A. R. F., \& Tabatabaei, S. M. A. (2013). Effect of tramadol at different doses on orthodontic tooth movement and bone resorption in rats. Dental Research Journal, 10(3), 337-42. http://drj.mui. ac.ir/index.php/drj/article/view/1455

Brown, E. D., Calhoun, N. R., Larson, R. H., \& Smith Jr, J. C. (1979). An effect of zinc deficiency on dental caries. Life Sciences, 24(22), 2093-97. [DOI:10.1016/0024-3205(79)90083-3]

Cerklewski, F. L. (1981). Effect of suboptimal zinc nutrition during gestation and lactation on rat molar tooth composition and dental caries. The Journal of Nutrition, 111(10), 1780-3. [DOI:10.1093/jn/111.10.1780] [PMID]

Chen, C. Y., \& Lin, K. M. (2009). Health consequences of illegal drug use. Current Opinion in Psychiatry, 22(3), 287-92. [DOI:10.1097/YCO.0b013e32832a2349] [PMID]

Friedler, G., \& Cicero, T. (1987). Paternal pregestational opiate expossure in male mice: Neuroendocrine deficits in their offspring. Research Communications in Substances of Abuse, 8(3-4), 109-16. [PMCID] [PMID]
Gibson, T. P. (1996). Pharmacokinetics, efficacy, and safety of analgesia with a focus on tramadol $\mathrm{HCl}$. The American Journal of Medicine, 101(1A), S47-S53. [DOI:10.1016/S00029343(96)00138-6

Gupta, A. (2018). Comprehensive biochemistry for dentistry: textbook for dental students. Berlin: Springer. [DOI:10.1007/978-981-131035-5]

Henkel, K., Altenburger, M. J., Auwärter, V., \& Neukamm, M. A. (2018). Full validation of a method for the determination of drugs of abuse in non-mineralized dental biofilm using liquid chromatography-tandem mass spectrometry and application to postmortem samples. Talanta, 176, 360-366. [DOI:10.1016/j. talanta.2017.08.047] [PMID]

Larson, R. H. (1964). Effect of prenatal nutrition on oral structures. Journal of the American Dietetic Association, 44, 368-73. [PMID]

Lhamo, Y., Chugh, P. K., Gautam, S. R., \& Tripathi, C. D. (2017). Epidemic of Vitamin D deficiency and its management: awareness among indian medical undergraduates. Journal of Environmental and Public Health, 2017, 2517207. [DOI:10.1155/2017/2517207] [PMID] [PMCID]

Lin, C. Y., Lin, H. H., Tsai, M. H., Lin, S. P., \& Chen, M. H. (2011) Zinc chloride for odontogenesis of dental pulp stem cells via metallothionein up-regulation. Journal of Endodontics, 37(2), 211-6. [DOI:10.1016/j.joen.2010.11.009] [PMID]

Man, R. E. K., Li, L. J., Cheng, C. Y., Wong, T. Y., Lamoureux E., \& Sabanayagam, C. (2017). Prevalence and determinants of suboptimal vitamin D levels in a multiethnic Asian population. Nutrients, 9(3), 313. [DOI:10.3390/nu9030313] [PMID] [PMCID]

Nadeau, R., Hasstedt, K., Sunstrum, A. B., Wagner, C., \& Tu, H. (2018). Addressing the opioid epidemic: Impact of opioid prescribing protocol at the University of Minnesota School of Dentistry. Craniomaxillofacial Trauma \& Reconstruction, 11(2), 104-10. [DOI:10.1055/s-0038-1649498] [PMID] [PMCID]

Navia, J. M., \& JM, N. (1972). Prevention of dental caries: Agents which increase tooth resistance to dental caries. International Dental Journal, 22(4), 427-40. [PMID]

Navia, J. M., Di Orio, L. P., Menaker, L., \& Miller, S. (1970). Effect of undernutrition during the perinatal period on caries development in the rat. Journal of Dental Research, 49(5), 1091-8. [DO I:10.1177/00220345700490051601] [PMID]

Nessa, A., Latif, S. A., Siddiqui, N. I., Hussain, M. A., \& Hossain, M. A. (2008). Drug abuse and addiction. Mymensingh Medical Journal, 17(2), 227-35.

Rashidpour, M., Akhoundi, M. A., Nik, T. H., Dehpour, A. R. Alaeddini, M., \& Javadi, E., et al. (2012). Effect of Tramado ( $\mu$-opioid receptor agonist) on orthodontic tooth movements in a rat model. Journal of Dentistry (Tehran, Iran), 9(2), 83-9. [PMCID] [PMID]

Reece, A. S. (2007). Dentition of addiction in Queensland: poor dental status and major contributing drugs. Australian Dental Journal, 52(2), 144-9. [DOI:10.1111/j.1834-7819.2007.tb00480.x] [PMID]

Robinson, P. G., Acquah, S., \& Gibson, B. (2005). Drug users: Oral health-related attitudes and behaviours. British Dental Journal, 198(4), 219-24. [DOI:10.1038/sj.bdj.4812090] [PMID] 
Scheutz, F. (1984). Five-year evaluation of a dental care delivery system for drug addicts in Denmark. Community Dentistry and Oral Epidemiology, 12(1), 29-34. [DOI:10.1111/j.1600-0528.1984. tb01405.x] [PMID]

Sebastiani, G., Borrás-Novell, C., Alsina Casanova, M., Pascual Tutusaus, M., Ferrero Martínez, S., \& Gómez Roig, M. D., et al. (2018). The effects of alcohol and drugs of abuse on maternal nutritional profile during pregnancy. Nutrients, 10(8), 1008. [DOI:10.3390/nu10081008] [PMID] [PMCID]

Sekhavat, A. R., Mousavizadeh, K., Pakshir, H. R., \& Aslani, F. S. (2002). Effect of misoprostol, a prostaglandin E1 analog, on orthodontic tooth movement in rats. American Journal of Orthodontics and Dentofacial Orthopedics, 122(5), 542-7.[DOI:10.1067/ mod.2002.126153] [PMID] 